\title{
Haack, Adrian (2018). Dramapädagogik, Selbstkompetenz und Professionalisierung. Performative Identitätsarbeit im Lehramtsstudium Englisch. Wiesbaden, J. B. Metzler/Springer
}

\author{
Michael K. Legutke
}

Obwohl die Erkenntnis nicht neu ist, dass es nicht zuletzt auf die Lehrerin, auf den Lehrer ankommt, wenn man nach Gründen für den Erfolg des Fremdsprachenunterrichts fragt, hat die fremdsprachendidaktische Forschung im deutschsprachigen Raum Aspekte der Professionalisierung von Lehrer*innen lange Zeit bestenfalls als Nebenschauplatz behandelt (vgl. Legutke \& Schart 2016, Roters \& Trautmann 2014). Besonders die erste Phase der Lehrer*innenbildung wurde lange Zeit vernachlässigt. Erst in jüngerer Zeit schalten sich Fremdsprachendidaktiker*innen verstärkt forschungsbasiert in die Professionalisierungsdiskussion ein. Die umfangreiche Studie von Adrian Haack ist in diesem hoch relevanten Forschungsfeld verortet. Der Verfasser stellt sich der anspruchsvollen Aufgabe, die subjektive Dimension professioneller Kompetenz zu beschreiben und Wege zu erörtern, wie diese bereits in der ersten Phase der Ausbildung entwickelt werden kann. Schlüsselkonzept der Studie ist die Selbstkompetenz der Lehrperson, die, so Haacks Ausgangsthese, durch den Einsatz dramapädagogischer Verfahren in der universitären Ausbildung entscheidende Entwicklungsimpulse erhalten kann.

Die Arbeit beginnt (Kap. 1) mit einem engagierten und gut begründeten Plädoyer, den individuellen Personen und Persönlichkeiten der Lehramtsstudierenden, insbesondere in der universitären Phase der Ausbildung, einen größeren Stellenwert zukommen zu lassen als das in der Vergangenheit der Fall war. Die Lehrerbildung müsse sich sehr viel intensiver mit den Selbstbildern zukünftiger Lehrkräfte befassen und Anstöße für Selbsterfahrungsarbeit geben, damit Studierende folgende Schlüsselfragen in den Blick nehmen: Wer bin ich? Wie sehe ich meine Rolle als Lehrer, als Lehrerin? Was für ein Lehrer, was für eine Lehrerin will ich sein? Besonders Fremdsprachenlehrer*innen müssten „sich sehr bewusst über ihr ,Wer" sein, das sie Schüler*innen gegenüber darstellen“ (6). Diese Forderung ist nicht neu (vgl. z.B. Schewe 1993, Kortmann 2004), aber höchst relevant (Legutke \& Schart 2016). Drama- und theaterpädagogische Ansätze als integrale Bestandteile akademischer Lehrerbildung, so die zentrale These Haacks, bieten nicht nur den für die Selbsterfahrungsarbeit notwendigen Handlungsraum, sondern haben zugleich einen hohen Transferwert für einen lebendigen und kommunikativen Fremdsprachenunterricht. Die folgenden 
Teile der Studie entfalten diese These in zwei großen Blöcken. Der erste umfasst eine differenzierte Erörterung des zentralen Konstrukts der "Selbstkompetenz“ in seinem Verhältnis zum Konzept der „Identität“ (Kap. 2), eine kompakte Einführung in die Arbeitsweisen der Dramapädagogik (Kap. 3) und schließlich mit 82 Seiten das Herzstück dieses Blocks, eine sehr kenntnisreiche und brillant dargestellte Entwicklung eines Fördermodells von professioneller Selbstkompetenz durch dramapädagogische Lehrerbildung (4). Der zweite Block enthält dann die Darstellung und empirische Validierung von drei Typen dramapädagogischer Blockseminare, die der Verfasser über einen Zeitraum von mehreren Jahren im englischen Seminar der Universität Göttingen unterrichtete. Beide Blöcke sollen im Folgenden vorgestellt und erörtert werden.

Es gelingt dem Verfasser, ein begrifflich vielschichtiges und diffuses Feld überzeugend zu ordnen, indem er eine handhabbare und differenzierte Definition der Selbstkompetenz von Fremdsprachenlehrer*innen erarbeitet, deren Entwicklung bereits im Studium angestoßen werden sollte. Er beruft sich dabei auf ausgewählte Modelle der Pädagogik und der Humanistischen Psychologie, aus deren Synthese er vier Dimensionen eines professionellen Selbst bestimmt, die in ihrem komplexen Zusammenspiel die Lehreridentität ausmachen. Er unterscheidet das Selbst als Akteur, das Selbst als Erzählung, das dispositionale und motivationale Selbst sowie das Selbst als Lerner. So überzeugend Haacks Auswahl der Bezugskonzepte und so stimmig seine Argumentation ist, so bleibt dennoch zu fragen, weshalb er die motivationspsychologischen Ansätze Dörnyeis nicht berücksichtig hat, die u.a. das Selbstkonzept von Fremdsprachenlehrer*innen in Hinblick auf ihre Wertvorstellungen und Visionen erörtern (u.a. Dörnyei \& Kubaniova 2014). Hier hätte der Verfasser eine weitere fachspezifische Fundierung seines Fördermodells gefunden. Letzteres zeichnet sich dadurch aus, dass die genannten Dimensionen des Selbst mit dramapädagogischen Erfahrungsfeldern und einzelnen Bausteinen dramapädagogischer Arbeit verknüpft werden. Das Spektrum reicht von Körperarbeit, Schauspiel und Inszenierung über Imagination bis hin zu gruppen- und personenzentrierter Seminararbeit. In diesen Erfahrungsfeldern bieten sich den Studierenden vielfältige Möglichkeiten zur rationalen wie emotionalen Selbstwahrnehmung, zur Reflexion eigener und fremder Verhaltensweisen und zur narrativen Gestaltung beruflicher Selbstbilder. Hier können die Entwicklungsimpulse für den Erwerb des professionellen Selbst werdender Fremdsprachenlehrer*innen ihr Potenzial entfalten. Dass die differenzierte Erörterung des Fördermodells so anregend und lesenswert ist, liegt nicht nur daran, dass sie theoretisch vorzüglich fundiert ist, sondern dass sie von profunder Erfahrung Haacks mit Theaterarbeit zeugt. Schließlich stellt sich Haack zu jedem Teilaspekt seines Fördermodells die Frage, wie die in den Erfahrungsfeldern eingesetzten Verfahren auf den Fremdsprachenunterricht übertragen werden können. Er markiert damit die fremdsprachendidaktische Relevanz der „dramapädagogischen Selbstkompetenzarbeit".

Adrian Haack eröffnet den zweiten Block seiner Studie mit der Vorstellung der bereits erwähnten dramapädagogisch gestalteten Seminartypen für die 
Ausbildung von Fremdsprachenlehrer*innen. Mit Bezug auf das Fördermodell bietet er für jeden der drei Typen Hypothesen an, in welcher Weise dieser die Förderung von Selbstkompetenz voranbringen könne. Im Seminartyp I entwickeln Studierende ein Theaterstück in englischer Sprache, das sie in Praxisprojekten mit Schüler*innen inszenieren. Hier vermutet Haack, dass schon die kooperative Stückentwicklung und noch mehr die Inszenierungsarbeit mit den Schüler*innen von den Studierenden Offenheit, Flexibilität und die Fähigkeit, mit eigenen Emotionen und Erwartungen umzugehen, erfordert und somit wichtige Teilaspekte von Selbstkompetenz erfahrbar machen. Während durch den Seminartyp I Aspekte von Selbstkompetenz eher indirekt gefördert werden, ist der Seminartyp II explizit als „dramapädagogisches Selbstkompetenzseminar" gestaltet, das sich einer Vielzahl soziometrischer Methoden, Methoden der Selbsterfahrung, Methoden der Fantasiearbeit, des Erzähltheaters und Lehrerrollenspiels bedient. Seminartyp III schließlich wird als Regiekurs gestaltet, der an einem lokalen Theater stattfindet. Er endet mit einer Werkschau, für welche die Nachwuchsregisseur*innen eine Szene mit Laienschauspieler*innen inszenieren. Für alle drei Typen werden Ablaufprozesse und mögliche Aufgabenstellungen konkretisiert, die aus der reichen Erfahrung des Verfassers hervorgehen und die ein sehr lebendiges Bild einer möglichen Seminarpraxis entstehen lassen. Dramapädagogisch arbeitende Leser*innen werden hier vielfältige Anregungen für die eigene Praxis finden.

Adrian Haack hat seine Seminarpraxis über die Jahre forschend begleitet. Als Vertreter „praxisbezogener Forschung“ galt sein Interesse der Frage, in welchem Ausmaß die Seminare „Entwicklungsprozesse bezüglich der Selbstkompetenz der werdenden Fremdsprachenlehrer*innen anstoßen konnten“ (201). Zugleich ging es ihm darum, „ein tieferes Verständnis [. . .] der Selbstkompetenzentwicklungsprozesse werdender Lehrer*innen im Rahmen bestimmter Seminarformate" zu erlangen (ibid.). Haack ist sich dabei seiner besonderen Doppelrolle als Lehrender und zugleich Forschender bewusst. Antworten auf diese Fragen suchte er einmal durch eine zeitlich nahe Evaluation seiner Seminare. Zu diesem Zweck setzte er Fragebögen (vor und unmittelbar nach den Seminaren) mit standardisierten und offenen Fragen ein, führte Gruppeninterviews durch und erstellte Prozessdokumentationen mit Hilfe von Videographie und Beobachtungsprotokollen. Darüber hinaus gelang es ihm, eine onlinebasierte Befragung 40 ehemaliger Lehramtsstudierender durchzuführen, die an dramapädagogischen Lehrveranstaltungen teilnahmen, mit dem Ziel, mögliche Langzeitwirkungen der Seminare zu erfassen. Datengewinnung, Datenaufbereitung und Analyse werden gut nachvollziehbar dargestellt. Ein vom Verlag bereit gestelltes Onlineangebot bietet Leser*innen die Möglichkeit, Forschungsinstrumente, Verfahren und Daten im Detail zur Kenntnis zu nehmen, was die Lektüre zusätzlich bereichert. In diesem Onlineangebot hätten auch einige der Schlüsselgraphiken, mit denen Haack Ergebnisse darstellt, zusätzlich abgelegt werden können, denn durch die Verwandlung der ursprünglich farbigen Graphiken in Schwarzweißdruck sind sie teilweise 
schwer bzw. kaum zu lesen.

Haacks Studie ist ein stilistisch gelungenes, engagiertes und sehr kenntnisreiches Plädoyer für eine Neuorientierung fremdsprachlicherLehrer*innenbildung, die die Person und ihre Entwicklung stärker ins Zentrum der Bemühungen rückt, indem sie dramapädagogische Elemente und Verfahren in Lehr- und Lernszenarien integriert. Die Studie ermöglicht nicht nur einen wertvollen und sehr praxisnahen Einblick in identitätsfördernde Arbeit mit Lehramtsstudierenden. Sie erbringt auch den Nachweis, dass die Hochschulseminare zumindest für die Gruppe der werdenden Lehrkräfte, die sich entschieden, die dramapädagogischen Seminare zu besuchen, die Entwicklung von Selbstkompetenz befördern. Die Seminartypen sind ohne Frage ein ernst zu nehmender Beitrag zur Professionalisierung, denn sie geben nicht zuletzt Entwicklungsanstöße für die Herausbildung eines reflektierenden Habitus.

Der Umfang der Studie mit 360 Seiten und 370 Fußnoten sollte Leser*innen nicht abschrecken, denn es erwies sich bei der Lektüre immer wieder als gewinnbringend, den Exkursen und Zusatzerklärungen in den Fußnoten $\mathrm{zu}$ folgen, auch wenn manche von ihnen besser gestrichen oder eher in den Haupttext integriert worden wären. Auch der Haupttext hätte an manchen Stellen deutlich gestrafft werden können. Gerade angesichts der Komplexität des Unternehmens und der vielfältigen Diskurse, auf die der Verfasser gekonnt und kenntnisreich Bezug nimmt, würde ein Sachindex das große Potential der Arbeit leichter erschließbar machen. Ein solcher Index fehlt aber leider. Nichtsdestotrotz lohnt sich die Lektüre für diejenigen, die sich um die Professionalisierung zukünftiger Fremdsprachenlehrer*innen bemühen und auf diesem Feld forschen. Besonders gewinnbringend ist die Studie für dramapädagogisch arbeitende Hochschullehrende. Es ist zu hoffen, dass die von Haack vorgenommene, hochschuldidaktische Positionierung von Ausbildungsforschung gebührend wahrgenommen und für andere Inhaltsfelder weiterentwickelt wird. Haack hat hier Pionierarbeit geleistet. Die Lektüre ist ferner vor allem auch Nachwuchsforscher*innen zu empfehlen, die ein Forschungsprojekt in der Doppelrolle als Forschende und Lehrende verfolgen. Ihnen kann Haacks innovative Studie als Referenzarbeit für das eigene Projekt dienen.

\section{Bibliografie}

Dörnyei, Zoltan, \& Kubanyiova, Magdalena (2014): Motivating learners, motivating teachers: Building vision in the language classroom. Cambridge: Cambridge University Press

Korthagen, Fred (2004): In search of the essence of a good teacher: towards a more holistic approach in teacher education. In: Teaching and Teacher Education 20, 77-97 
Legutke, Michael \& Schart, Michael (2016): Fremdsprachendidaktische Lehrerbildungsforschung. Bilanz und Perspektiven. In: Legutke, Michael \& Schart, Michael (Hrsg.). Fremdsprachendidaktische Professionsforschung: Brennpunkt Lehrerbildung. Tübingen: Narr, 9-46

Roters, Bianca \& Trautmann, Matthias (2014): Professionalität von Fremdsprachenlehrenden. Theoretische Zugänge und empirische Befunde. In: Fremdsprachen Lehren und Lernen 43/1, 51-65

Schewe, Manfred (1993): Fremdsprache inszenieren. Zur Fundierung einer dramapädagogischen Lehr- und Lernpraxis. Oldenburg: Universität Oldenburg, Zentrum für pädagogische Berufspraxis 